\title{
Changes in serum biomarker profiles after percutaneous mitral valve repair with the MitraClip system
}

Ji-Na Yoon ${ }^{1 *}$, Antonio H. Frangieh ${ }^{1 *}$, Adrian Attinger-Toller ${ }^{1}$, Christiane Gruner ${ }^{2}$,

Felix C. Tanner ${ }^{2}$, Maurizio Taramasso ${ }^{3}$, Roberto Corti ${ }^{1 \#}$, Thomas F. Lüscher ${ }^{1}$, Frank Ruschitzka ${ }^{4}$, Dominique Bettex ${ }^{5}$, Francesco Maisano ${ }^{3}$, Oliver Gaemperli ${ }^{1}$

${ }^{1}$ Andreas Grüntzig Cardiac Catheterization Laboratories, University Heart Center, Zurich, Switzerland

${ }^{2}$ Echocardiography, University Heart Center, Zurich, Switzerland

${ }^{3}$ Cardiac Surgery, University Heart Center, Zurich, Switzerland

${ }^{4}$ Heart Failure Clinic, University Heart Center, Zurich, Switzerland

${ }^{5}$ Cardiac Anesthesiology, University Hospital Zurich, Switzerland

\begin{abstract}
Background: Mitral regurgitation (MR) is one of the most common valvular diseases. Percutaneous mitral valve repair with the MitraClip ${ }^{T M}$ system is a novel percutaneous mitral valve repair (PMVR) technique for high-surgical-risk patients. However, the effect of PMVR on circulating cardiac or inflammatory biomarkers and their association with individual functional, echocardiographic and clinical outcomes is poorly investigated.

Methods: A group of 144 patients with functional or degenerative MR (age, $75 \pm 11$ years; $41 \%$ females) underwent PMVR with the MitraClip system at the University Heart Center Zurich. Serum biomarkers as $N$-terminal pro-B-type natriuretic peptide (NT-proBNP), C-reactive protein (CRP) and creatinine were obtained from venous sampling at baseline and follow-up of 3-6 months.

Results: Median NT-proBNP decreased insignificantly from 2,942 (IQR 1,596-5,722) to 2,739 (IQR 1,440-4,296) $n g / L, p=0.21$. NT-proBNP changes did not correlate with baseline left ventricular (LV) ejection fraction or LV dimensions, with New York Heart Association class on follow-up, or with clinical events on follow-up. CRP levels reached a peak on the third postoperative day at $34.0 \mathrm{mg} / \mathrm{L}$ with a subsequent slow decrease over the ensuing days.

Conclusions: Despite successful PMVR, NT-proBNP remain fairly unchanged on follow-up and changes in NT-proBNP levels are poor predictors of functional improvement or clinical outcome after MitraClip treatment. (Cardiol J 2016; 23, 4: 384-392)
\end{abstract}

Key words: MitraClip, mitral regurgitation, percutaneous mitral valve repair, serum biomarkers, NT-proBNP

Address for correspondence: Oliver Gaemperli, MD, Andreas Grüntzig Laboratories And Cardiac Imaging,

University Heart Center, Raemistrasse 100, 8091 Zurich, Switzerland, tel: +41 44255 1052, fax: +41 442554401 ,

e-mail: oliver.gaemperli@usz.ch

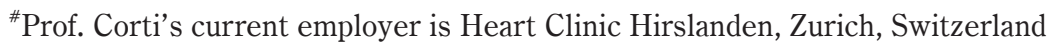

*Contributed equally to this work

Received: 11.04.2016

Accepted: 24.05.2016 


\section{Introduction}

Mitral regurgitation (MR) is the most common valve pathology found in the general population. Recently, percutaneous edge-to-edge mitral valve repair (PMVR) using the MitraClip ${ }^{\mathrm{TM}}$ system has emerged as a valuable alternative to surgery in high-risk patients with anatomical suitability for the device. First human trials, including the Endovascular Valve Edge-to-Edge Repair Study I (EVEREST), documented safety, reduction in MR, reverse left ventricular (LV) remodeling and improvements in quality of life $[1,2]$. The EVEREST II randomized trial compared the safety and efficacy of the MitraClip device to mitral valve surgery in low-risk surgical candidates and demonstrated slightly lower efficacy but lower complication rates [3]. Moreover, retrospective studies suggest better outcomes after PMVR in high-risk patients compared to medical therapy [4].

Cardiac biomarkers have become integral elements in the clinical assessment and management of patients with cardiac disease. Their levels usually correlate closely with disease severity and prognosis. B-type natriuretic peptide (BNP), a cardiac neurohormone, has vasodilating effects and relaxes vascular smooth muscle cells causing peripheral veno- and vasodilatation, and counteracting the high LV wall stress by reducing pre- and afterload. Especially in patients with heart failure (HF), this neurohormone is released in the presence of volume or pressure overload of the ventricles in response to an increase in wall tension [5]. The 76-residue N-terminal fragment proBNP (NT-proBNP) has a longer half-life (120 $\mathrm{min})$ and a more stable constitution than BNP, and therefore, it is less dependent on short fluctuations in volume status [6, 7]. PMVR with use of the MitraClip device can reduce $\mathrm{LV}$ wall stress by reducing MR and thereby induce reverse remodeling of both ventricles with reduction in diastolic and systolic LV volumes, however large studies assessing biomarker changes are scarce [8-11]. On the other hand, C-reactive protein (CRP) plays an important role in systemic response to inflammation. Several scientific reports have been published, highlighting the clinical use of CRP in diagnosis and prognosis of congestive HF [12]. An increase in CRP values in the early postoperative period after transcatheter aortic valve implantation (TAVI) was shown [13]. However, data on CRP changes after PMVR have been missing.

Thus, the aim of the present study was to evaluate the effect of PMVR on circulating cardiac or inflammatory biomarkers (NT-pro BNP and CRP), and their association with individual functional, echocardiographic and clinical outcomes.

\section{Methods}

\section{Study design}

This study is a retrospective single-center observational clinical study of 144 patients with moderate-to-severe MR, who received PMVR using the MitraClip system at the University Heart Center Zurich. Patients were included in the prospective MitraSwiss registry (MitraSwiss registry, KEK-ZH-Nr. 2010-0466), which is a national investigator-driven multicenter clinical registry that includes clinical, echocardiographic and biomarker follow-up of the patients. The study protocol was approved by the local institutional review board and all patients gave a written informed consent for their inclusion in the registry (MitraSwiss registry, KEK-ZH-Nr. 2010-0466).

\section{Patient population}

The severity of MR was assessed with two-dimensional (2D) echocardiography according to the recommendations of the American Society of Echocardiography [14]. Indications for PMVR included patients with symptomatic $\mathrm{MR}(\geq 3+)$ due to degenerative or functional MR. High-surgicalrisk status defined by the European System for Cardiac Operative Risk Evaluation (EuroSCORE) was an additional criterion for PMVR. An interdisciplinary team of interventional cardiologists, echocardiographers, cardiac anesthetists, and cardiac surgeons from the University Heart Center Zurich selected patients for PMVR. Patients with rheumatic heart disease, endocarditis, a mitral valve orifice area $\leq 2.0 \mathrm{~cm}^{2}$, extensive prolapse of flail leaflets (prolapse width $>25 \mathrm{~mm}$, flail gap $>20 \mathrm{~mm}$ ) or patients who had any interventional or surgical procedure within 30 days were excluded from treatment [15].

\section{Percutaneous mitral valve repair procedure}

Percutaneous mitral valve repair with the MitraClip device (Abbott Vascular Structural Heart, Menlo Park, CA) was performed according to standard technique described elsewhere [15] with echocardiographic (3D transesophageal echocardiography) and fluoroscopic guidance, and under general anesthesia. In the case of suboptimal reduction of MR, a second clip or third clip can be inserted on the lateral or medial side from the first clip. Acute procedural success was defined as suc- 
cessful MitraClip implantation with immediate MR reduction to grade $2+$ or less by echocardiography.

\section{Laboratory data}

For this study, non-cardiac-specific and cardiacspecific laboratory data were obtained by venous sampling through clinical follow-up at 3, 6 and 12 months after PMVR in generally stable patients. NT-proBNP belongs to cardiac-specific biomarkers. Non-cardiac specific biomarkers were creatinine and CRP that was additionally measured in the early postoperative setting and during the same hospitalization to assess early postoperative inflammatory response. Hematology values like hemoglobin and platelet count were also collected. Biomarker values from patients with clinical signs and symptoms of overt fluid retention or admitted for decompensated $\mathrm{HF}$ were rejected from the analysis.

\section{Follow-up}

Clinical follow-up was obtained from clinical records and by directly contacting the patients' general physician. Clinical endpoints included death, reoperation of the mitral valve, hospitalization for HF and heart transplantation. New York Heart Association (NYHA) functional class was obtained on clinical follow-up at 3-6 months.

\section{Statistical analysis}

All analyses were performed using SPSS version 20.0 for Windows (SPSS, Inc., Chicago, Illinois). Quantitative data are expressed as mean \pm standard deviation (SD) or median with interquartile range (IQR) where appropriate, and categorical data given in proportions and percentages. Statistical comparison of quantitative data was performed using a 2-tailed Wilcoxon signed rank test (Mann-Whitney U test) for paired samples or Kruskal-Wallis test for unrelated samples. Categorical data was compared with Fisher's exact test. Results from different measurements were correlated using Spearman's method. To analyze the follow-up data we obtained cumulative event rates using the Kaplan-Meier function. Event-free survival curves were plotted for a composite endpoint of death, HF re-hospitalization, reoperation and heart transplantation, and compared using the log-rank test. A p value $<0.05$ was considered statistically significant for all tests.

\section{Results}

Patient characteristics and procedural data

A group of 144 patients had baseline and follow-up biomarkers available and were included
Table 1. Patients baseline characteristics $(n=144)$.

\begin{tabular}{lc}
\hline Age [years] & $75 \pm 11$ \\
Male gender & $85(59 \%)$ \\
Body mass index $\left[\mathrm{kg} / \mathrm{m}^{2}\right]$ & $25 \pm 5$ \\
Clinical features: & \\
Arterial hypertension & $100(69 \%)$ \\
Hyperlipidemia & $60(42 \%)$ \\
Diabetes mellitus & $26(18 \%)$ \\
Coronary artery disease: & $64(44 \%)$ \\
$\quad$ Previous MI & $36(25 \%)$ \\
$\quad$ Previous PCI & $44(31 \%)$ \\
$\quad$ Previous CABG & $21(15 \%)$ \\
Left ventricular ejection fraction [\%] & $46 \pm 18$ \\
Previous valve surgery & $13(9 \%)$ \\
Previous vascular/thoracic surgery & $4(3 \%)$ \\
Atrial fibrillation & $136(94 \%)$ \\
COPD & $21(15 \%)$ \\
Previous stroke & $14(8 \%)$ \\
Impaired renal function & $87(60 \%)$ \\
Cancer & $15(10 \%)$ \\
NYHA functional class: & \\
II & $17(12 \%)$ \\
III & $99(69 \%)$ \\
IV & $28(19 \%)$ \\
\hline
\end{tabular}

Data are given as mean \pm standard deviation unless otherwise stated. CABG - coronary artery bypass grafting; COPD - chronic obstructive pulmonary disease; $\mathrm{Ml}$ - myocardial infarction; NYHA - New York Heart Association; PCl — percutaneous coronary intervention

in this study. Baseline characteristics are shown in Table 1 . The mean age was $75 \pm 11$ years and 85 (59\%) participants were male patients. Most patients had comorbidities including arterial hypertension, hyperlipidemia, diabetes mellitus, or coronary artery disease. The mean LV ejection fraction was $46 \pm 18 \%$. Thirteen (9\%) patients had previous valve surgery and 4 (3\%) patients had previous vascular/thoracic surgery. One hundred and twenty-seven $(88 \%)$ patients were in NYHA-functional class III-IV. Most patients had history of atrial fibrillation and impaired renal function.

The etiology and mechanism of MR is shown in Table 2. A functional mechanism was identified in $80(56 \%)$ patients, while a degenerative etiology was seen in 55 (38\%) patients.

Acute procedural success was achieved in 136 (94\%) patients. The majority $(84 ; 58 \%$ patients) had 2 clips implanted, 37 (26\%) patients had 1 clip, 21 (15\%) patients had 3 clips and in the rare case of $2(1 \%)$ patients, there were 4 clips implanted. In-hospital death occurred in 6 (4\%) patients. 
Table 2. Etiology and mechanism of mitral regurgitation $(n=144)$.

\begin{tabular}{lc}
\hline Functional: & $80(56 \%)$ \\
Ischemic & $35(24 \%)$ \\
Non-ischemic & $45(32 \%)$ \\
Degenerative & $55(38 \%)$ \\
Mixed & $9(6 \%)$ \\
\hline
\end{tabular}

Data are given as absolute numbers and percentages

\section{Biomarker profiles}

Biomarker laboratory data were available at baseline (before the procedure) and at 3-6-month follow-up. NT-proBNP data were available from 91 patients, creatinine from 106 patients, creatinine-clearance from 82 patients, CRP from 73 patients, hemoglobin from 102 patients, and platelet counts from 98 patients, as shown in Table 3.

The median NT-proBNP level decreased slightly after mitral valve clipping from 2,942 (IQR 1,596$-5,722)$ to 2,739 (IQR 1,440-4,296) ng/L (Fig. 1). However, this difference fell short of statistical significance $(\mathrm{p}=0.21)$. Median change in pro-BNP levels was $-67 \mathrm{ng} / \mathrm{L}$, however with a large variation of values ranging from $-28,211 \mathrm{ng} / \mathrm{L}$ to $+11,217 \mathrm{ng} / \mathrm{L}$ (Fig. 2). CRP levels decreased insignificantly from 4.3 (IQR $1.0-12.0)$ to $3.7(1.2-10.0) \mathrm{mg} / \mathrm{L}$ $(\mathrm{p}=0.25)$. There was no significant change of creatinine level (from 118 to $120 \mu \mathrm{mol} / \mathrm{L} ; \mathrm{p}=0.72$ ) or creatinine-clearance (from 49 to $47 \mathrm{~mL} / \mathrm{min} ; \mathrm{p}=0.18$ ). However, a slight but significant change in hemoglobin level from 12.3 (IQR 10.8-13.4) to 12.4 (11.3-13.6) $\mathrm{g} / \mathrm{dL}$ was observed after the MitraClip procedure $(\mathrm{p}=0.026)$.

\section{NT-proBNP}

There was no significant correlation of changes in NT-proBNP with baseline LV ejection fraction $(\mathrm{r}=-0.37, \mathrm{p}=0.74), \mathrm{LV}$ end-diastolic volume $(\mathrm{r}=0.049, \mathrm{p}=0.68)$, or LV end-systolic volume $(\mathrm{r}=0.099, \mathrm{p}=0.41)$, however a weak, but sig-

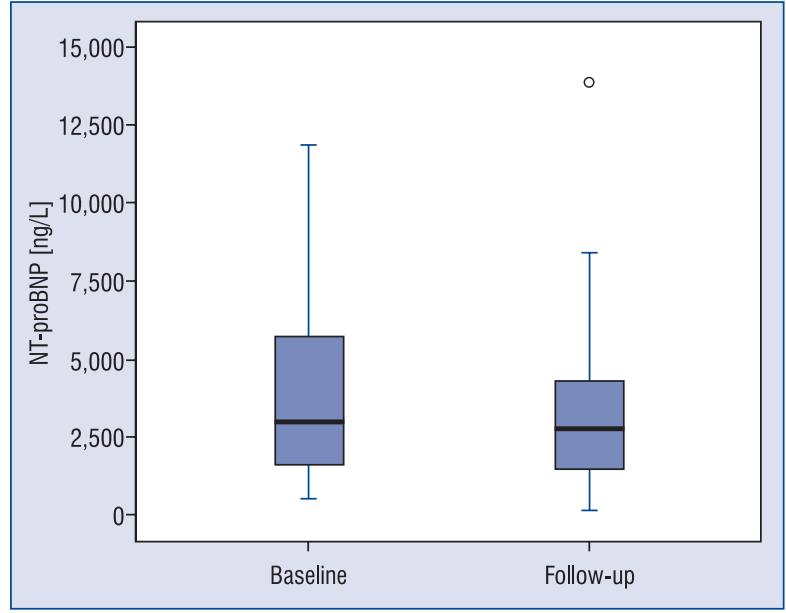

Figure 1. N-terminal pro-B-type natriuretic peptide (NT-proBNP) serum levels at baseline and follow-up (3-6 months) after MitraClip implantation given as boxplots with median value (black line), interquartile range (box), total range (whiskers) and outliers (circles).

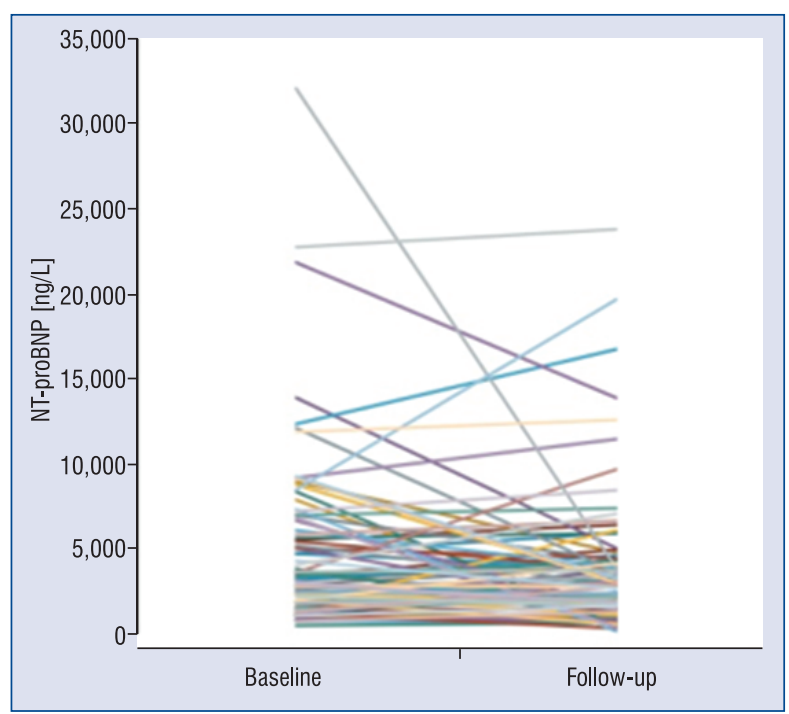

Figure 2. Individual changes in N-terminal pro-B-type natriuretic peptide (NT-proBNP) levels at baseline and follow-up after 3-6 months.

Table 3. Biochemical and hematological values before and after MitraClip implantation.

\begin{tabular}{lcccc}
\hline Parameter & N & Baseline & 3-6 month & P \\
\hline NT-pro BNP $[\mathrm{ng} / \mathrm{L}]$ & 91 & $2942(1596-5722)$ & $2739(1440-4296)$ & 0.21 \\
Creatinine $[\mu \mathrm{mol} / \mathrm{L}]$ & 106 & $118(89-156)$ & $120(91-149)$ & 0.72 \\
Cr-clearance $[\mathrm{mL} / \mathrm{min}]$ & 82 & $49(33-73)$ & $47(33-65)$ & 0.18 \\
C-reactive protein $[\mathrm{mg} / \mathrm{L}]$ & 73 & $4.3(1.0-12.0)$ & $3.7(1.2-10.0)$ & 0.25 \\
Hemoglobin $[\mathrm{g} / \mathrm{dL}]$ & 102 & $12.3(10.8-13.4)$ & $12.4(11.3-13.6)$ & 0.026 \\
Platelet count $\left[\times 10^{3} / \mu \mathrm{L}\right]$ & 98 & $225(181-270)$ & $220(169-258)$ & 0.88 \\
\hline
\end{tabular}

All values are given as median (interquartile range); NT-proBNP — N-terminal pro-B-type natriuretic peptide; $\mathrm{Cr}$-clearance — creatinine-clearance 


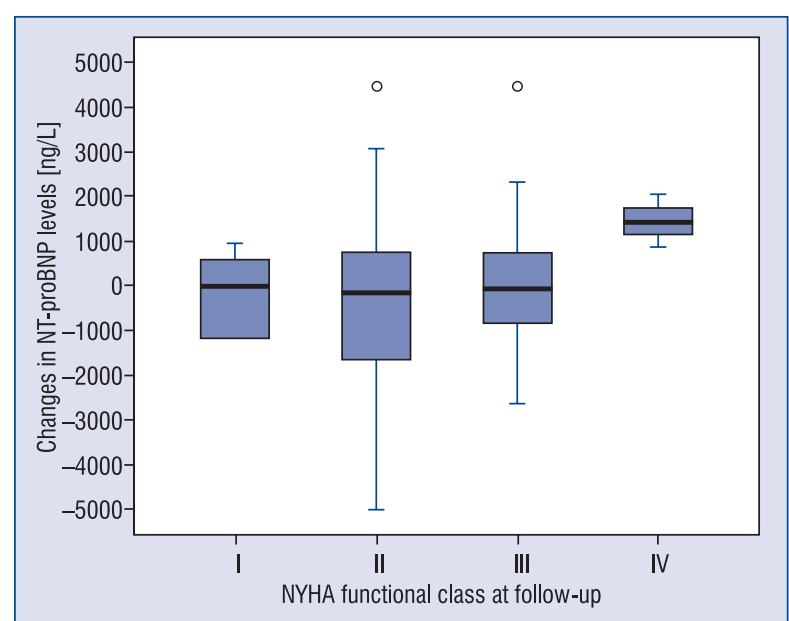

Figure 3. Changes in N-terminal pro-B-type natriuretic peptide (NT-proBNP) serum levels compared to the follow-up New York Heart Association (NYHA) classification given as boxplots with median value (black line), interquartile range (box), total range (whiskers) and outliers (circles).

nificant inverse correlation was found for baseline pulmonary pressures $(r=-0.32, p=0.007)$ and a weak positive correlation with the change in pulmonary pressure from baseline to $3-6$-month follow-up ( $r=0.35, p=0.006)$.

In patients with functional MR, median NT-proBNP level decreased from 3,195 (IQR 1,855$-5,828$ ) to 2,931 (IQR $1,608-4,787) \mathrm{ng} / \mathrm{L}$ after $3-6$-month follow-up $(\mathrm{p}=0.445)$. No significant correlation was found for the presence of functional MR and baseline NT-proBNP level $(r=-1.60$, $\mathrm{p}=0.110)$, although a significant correlation was found for follow-up NT-proBNP level $(\mathrm{r}=-2.313$, $\mathrm{p}=0.21$ ).

On the other hand, changes in NT-proBNP did not correlate with NYHA functional class at follow-up $(r=0.13, p=0.21)$. Figure 3 shows changes in NT-proBNP levels between baseline and follow-up (3-6 months) group based on NYHA functional class at follow-up. There was virtually no difference in median NT-proBNP changes in patients who were in NYHA class I, II, and III. Only in the worst group with NYHA class IV at followup, NT-proBNP increased by $1,414 \mathrm{ng} / \mathrm{L}$, however this change fell short of statistical significance compared to the other groups $(\mathrm{p}=0.21)$.

Median follow-up was 724 days (IQR 372$-1,073$ days). A combined endpoint occurred in $54(59 \%)$ out of 91 the patients. Event rates for a combined endpoint were similar between patients in which NT-proBNP values increased after Mi-

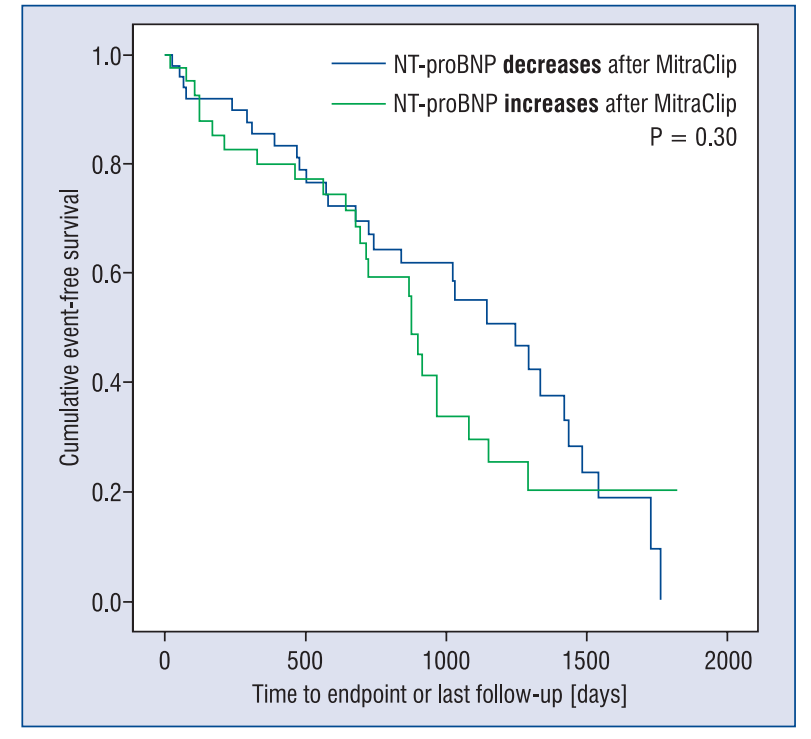

Figure 4. Survival of patients with an increase or decrease of N-terminal pro-B-type natriuretic peptide (NT-proBNP) is shown in the Kaplan-Meyer survival analysis. Combined endpoints were death, re-hospitalization for heart failure, reoperation and/or heart transplantation.

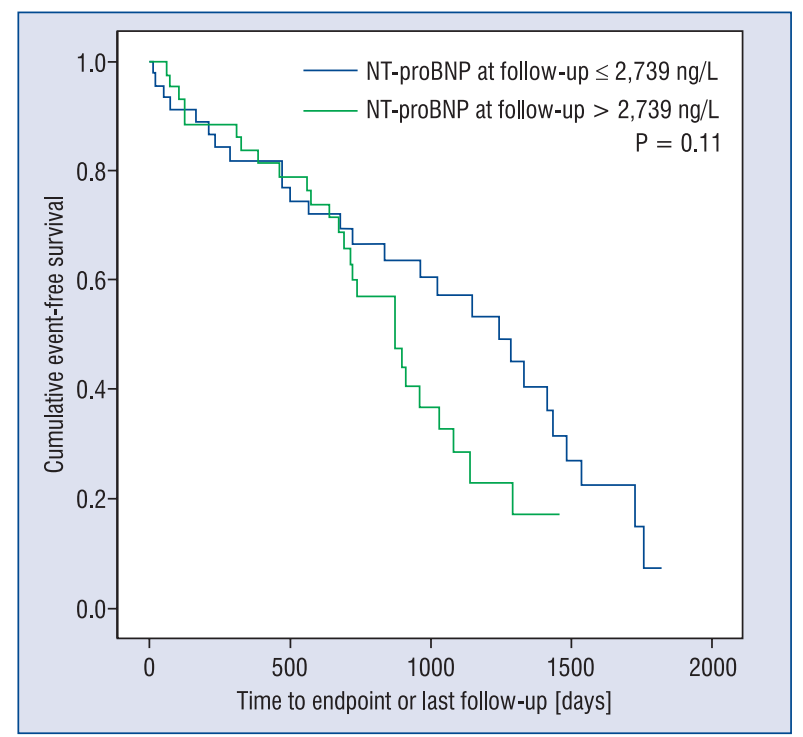

Figure 5. Survival of patients with absolute values of $\mathrm{N}$-terminal pro-B-type natriuretic peptide (NT-proBNP) at follow-up is shown as Kaplan-Meyer survival analysis. Combined endpoints for follow-up were death, rehospitalization for heart failure, reoperation and/or heart transplantation.

traClip implantation compared to those who had a decrease in NT-proBNP (Fig. 4). Similarly, absolute NT-proBNP values at follow-up appeared to be insignificant predictor of events (Fig. 5). Seven 


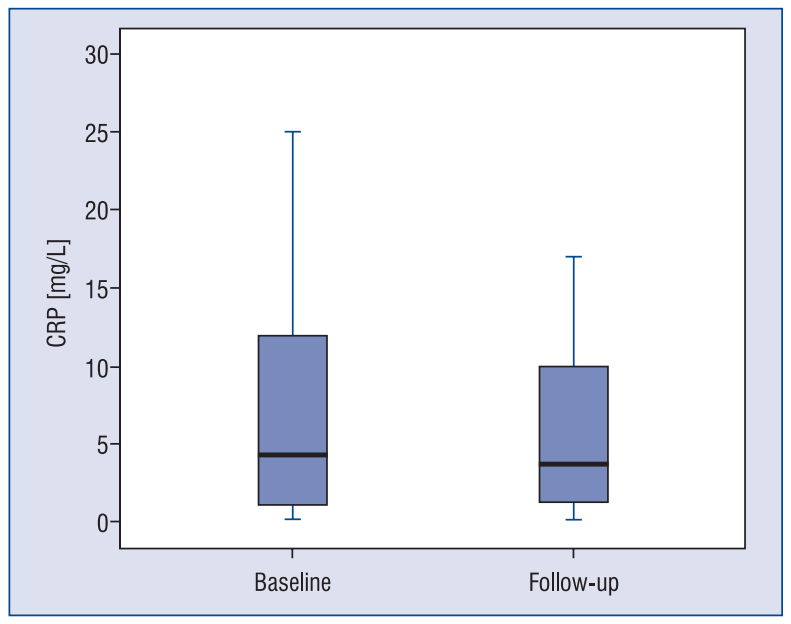

Figure 6. C-reactive protein (CRP) serum levels at baseline and follow-up (3-6 months) after MitraClip implantation given as boxplots with median value (black line), interquartile range (box), and total range (whiskers).

patients had NT-proBNP baseline levels $>10,000$ $\mathrm{ng} / \mathrm{L}$ of whom 4 patients reached a combined endpoint ( 1 death and 3 re-hospitalizations for HF).

\section{CRP}

Long-term CRP changes. CRP levels from 73 patients indicate numerical albeit statistically insignificant $(\mathrm{p}=0.25)$ decrease after PMVR (Fig. 6). Median change in CRP levels after MitraClip procedure was $-0.1 \mathrm{mg} / \mathrm{L}$ with a variation of values ranging from $-92 \mathrm{mg} / \mathrm{L}$ to $+37.4 \mathrm{mg} / \mathrm{L}$.
Short-term inflammatory response. Early postoperative CRP levels were available in 93 patients (Fig. 7). Median baseline CRP on the day of the PMVR procedure was $5.6 \mathrm{mg} / \mathrm{L}$ (IQR 1.1$-19.0 \mathrm{mg} / \mathrm{L}$ ) and increased over the ensuing 3 postoperative days reaching a peak on the $3^{\text {rd }}$ postoperative day at $34.0 \mathrm{mg} / \mathrm{L}$ (IQR $18.5-$ $-58.5 \mathrm{mg} / \mathrm{L}$ ). Thereafter, CRP levels regressed albeit at a lower rate with a median CRP of $18.5 \mathrm{mg} / \mathrm{L}$ (IQR $8.1-33.0 \mathrm{mg} / \mathrm{L}$ ) at day 7 .

\section{Discussion}

In our retrospective study of 144 patients with severe MR undergoing PMVR with the MitraClip system, we observed no statistically significant change in circulating biomarkers on long-term follow-up 3-6 months after successful PMVR. Median NT-proBNP decreased insignificantly and NT-proBNP changes did not correlate with either baseline LV ejection fraction or LV dimensions, nor NYHA class on follow-up, or with clinical events on follow-up. CRP levels reached a peak on the $3^{\text {rd }}$ postoperative day with a subsequent slow decrease over the ensuing days, and on follow-up, we showed numerical but insignificant decrease.

NT-proBNP serum levels reflect cardiac wall stress and higher concentrations can be measured in patients with HF [5]. MitraClip implantation can reduce $\mathrm{LV}$ wall stress by reducing $\mathrm{MR}$ and thereby induce reverse remodeling of both ventricles with reduction in diastolic and systolic LV volumes [8]. We assumed that NT-proBNP levels would sig-

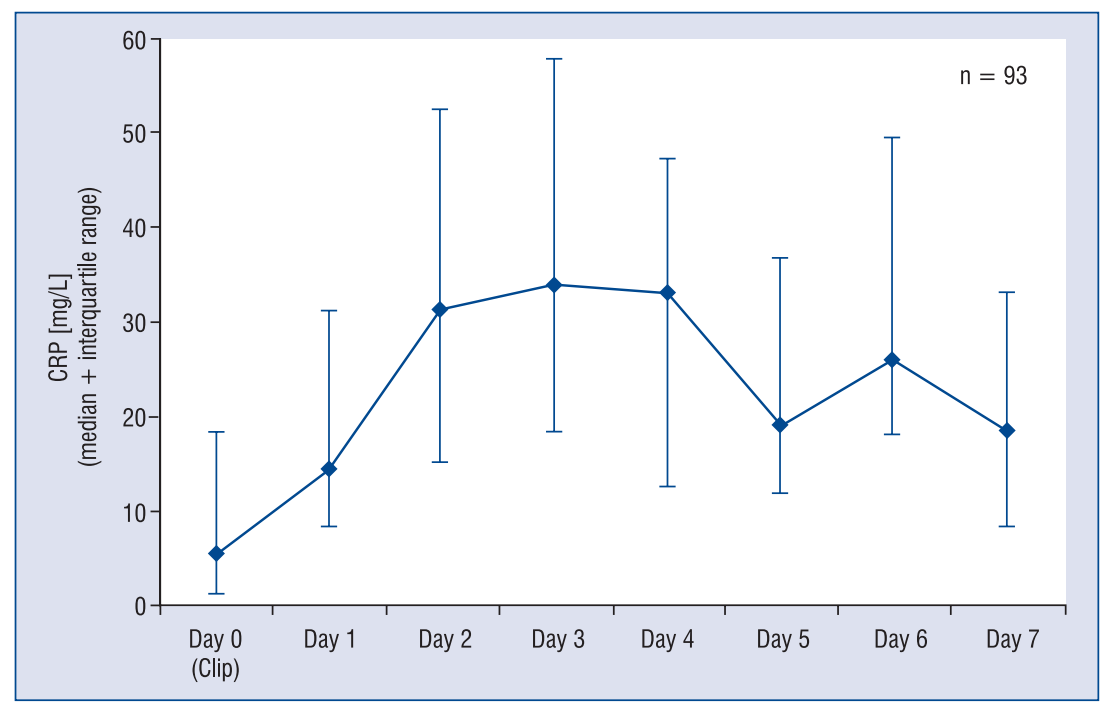

Figure 7. C-reactive protein (CRP)-course in the early postoperative period after percutaneous mitral valve repair with MitraClip as median value (bold blue line) with interquartile range (whiskers). 
nificantly decrease at 3-6-month follow-up when reverse remodeling starts to have an impact on the ventricles. However, our results show an insignificant reduction of NT-proBNP, similarly to a previously published sub-study from our group [16]. Interestingly, changes in NT-proBNP were similar in patients with functional MR versus degenerative MR. We expected changes in NT-proBNP to be less pronounced in patients with functional MR, since preload depends less on MR only, but also on the reduced contractility of the diseased myocardium. In addition, changes in NT-proBNP showed only a weak correlation with baseline pulmonary pressures and changes in pulmonary pressure after PMVR. Moreover, changes in NT-proBNP could not be predicted by baseline LV function or volumes. Changes in NT-proBNP were also a poor predictor for improvement of patients' HF symptoms after MitraClip implantation. Only patients with the poorest outcomes (NYHA class IV on follow-up) showed invariably an increase of NT-proBNP by $1,000 \mathrm{ng} / \mathrm{L}$ or more. However, similar postoperative NT-proBNP changes were observed for patients with follow-up NYHA class I, II, or III, respectively. Similarly, clinical event rates (death, re-hospitalization for HF, reoperation and/ /or heart transplantation) were similar in patients who decreased their NT-proBNP value compared to those in whom it increased.

In surgical mitral valve repair, there is an association between significant decrease of NT-proBNP levels and an improvement in HF symptoms [17]. Our data could not confirm this association, although generally there was a significant decrease in NYHA class in $63(70 \%)$ patients.

Thus, we conclude that serum NT-proBNP is marginally beneficial in the follow-up of $\mathrm{HF}$ patients after MitraClip implantation and should not be routinely performed to assess the clinical success of MitraClip implantation. A possible explanation of these findings lies in the underlying pathophysiological changes observed in the LV after PMVR - successful PMVR results in a significant decrease in LV preload from removing the regurgitant blood volume. These changes, however, are counteracted by a significant increase in afterload, which occurs from removing the low impedance pathway to LV systolic unloading. Since both, afterload and preload, appear to change to the same extent after PMVR, the net effect on LV wall stress (which consists of diastolic and systolic wall stress) reaches equipoise, and therefore NT-proBNP values do not change significantly [18].
Nonetheless, other hemodynamic studies have proven that despite these changes in afterload, PMVR improves hemodynamic profiles through an increase in cardiac index and a decrease in pulmonary pressures $[15,18]$.

Our results are somewhat at variance with NT-proBNP data reported in other studies, where a statistical significant decrease of NT-proBNP was reported [8-11]. Franzen et al. [11, 19] report a significant reduction in NT-proBNP values from $4,900 \mathrm{ng} / \mathrm{L}$ to $3,300 \mathrm{ng} / \mathrm{L}$ in 18 patients with endstage HF. The PERMIT-CARE registry of nonresponders to cardiac resynchronization therapy (CRT) reports a baseline NT-proBNP of 3,702 ng/L but no biomarker follow-up was obtained in this registry [20]. A very similar study by Seifert et al. [10] in 42 CRT non-responders, reported a reduction in NT-proBNP levels from 3,923 ng/L to 2,636 ng/L. Neuss et al. [9] followed 111 patients with congestive HF (73\% functional MR, ejection fraction $41 \%$ ) after PMVR and found a significant decrease in NT-proBNP from 3,452 ng/L to approximately $2,500 \mathrm{ng} / \mathrm{L}$. However, none of the large registries (ACCESS-Europe, TRAMI, Pilot European Sentinel, EVEREST High Risk Registry, and REALISM) included NT-proBNP as an endpoint.

Most studies with NT-proBNP data had a smaller number of patients ( $<35$ patients) than our study, except of Neuss et al. [9] with 111 patients. Additionally, baseline NT-proBNP values in the aforementioned trials were higher than in our patients, which may explain the significant reduction observed in those trials, and may make these trials more susceptible to statistical "regression to the mean" bias. In contrast, our patients had slightly lower baseline NT-proBNP values and higher ejection fraction, which may have blunted any potential effect of PMVR on changes in NT-proBNP.

Regarding survival analysis, previous studies showed that higher NT-proBNP levels had significantly poorer outcomes [9]. Our survival analysis could not confirm this statement. However, for patients at the extreme high range of NT-proBNP values with over $>10,000 \mathrm{ng} / \mathrm{L}$ it was more likely to have a poorer outcome. Although our study failed to prove NT-proBNP as a reliable biomarker, we recognize the potential advantages of additional serial measurements of NT-proBNP during longerterm follow-up. Nevertheless, the clinical condition of the patient and echocardiographic information seem to be more reliable parameters for follow-up shortly after MitraClip implantation. 
Similar to NT-proBNP, there was a numerical however non-statistical decrease of CRP level at long-term follow-up after 3-6 months. During the short-time follow-up, systemic inflammation reached a maximum on day 3 (median CRP level of $34.0 \mathrm{mg} / \mathrm{L}$; IQR $18.5-58.5 \mathrm{mg} / \mathrm{L}$ ) and decreased after a few days. CRP changes following MitraClip have been poorly investigated, in contrary to TAVI, where an increase in CRP values in the early postoperative period was shown, and was correlated with poorer outcome [13]. In comparison to TAVI, increases in post-procedural CRP levels after MitraClip implantation are 2-3 times lower [13]. The TAVI procedure is accomplished in majority of cases by retrograde transfemoral approach to the aortic valve, where a prosthetic stent expands the annulus of the aortic valve, thereby compressing the native valve [21]. However, PMVR with the MitraClip system, besides the transseptal puncture and the introduction of the $22 \mathrm{~F}$ steerable guide catheter, induces only a small local inflammation from smaller punctures and clip attachment on smaller parts of the leaflets, thus it is less traumatic and has fewer serious complications. This may explain the much smaller increase in CRP values in the early postoperative period.

\section{Limitations of the study}

The retrospective design of the study may be perceived as a limitation. As a consequence, there was no uniform prospective protocol for venous blood sampling available, and therefore biomarker documentation and follow-up was incomplete throughout our study population. Additionally, we could not obtain more sophisticated biomarkers of inflammation, such as interleukins or tumornecrosis factor.

The limited sample size is another important limitation of this study. It may well be that numerical differences observed in our sample would reach statistical significance if the sample was increased to several hundreds of patients. Nonetheless, statistical significance does not necessarily beget clinical significance. Therefore, we doubt that we would come to a very different clinical conclusion by a largely increased study population.

B-type natriuretic peptide values have a short half-life and their serum concentration varies according to volume-loaded conditions. This is why we chose NT-proBNP, which has a longer half-life and is less susceptible to short-term changes in volume conditions. Additionally, biomarker values were excluded if the patient was admitted to the hospital for HF or had obvious clinical signs of new fluid retention. However, despite these theoretical advantages of NT-proBNP, some marginal effects of volume status on serum levels cannot be denied. This study assessed only follow-up data at 3-6 months due to lack of laboratory data. NT-proBNP values after a longer follow-up may show different results, because reverse remodeling of the LV is an ongoing process.

\section{Conclusions}

Despite successful PMVR, NT-proBNP and CRP levels remain fairly unchanged on follow-up and changes in NT-proBNP are poor predictors of functional improvement or clinical outcome after MitraClip treatment.

We conclude that serum NT-proBNP has a limited clinical value in the follow-up of HF patients after MitraClip implantation. Other parameters, such as clinical and echocardiographic assessment should be prioritized over biomarkers to assess the long-term success of MitraClip implantation.

\section{Conflict of interest: None declared}

\section{References}

1. Feldman T, Kar S, Rinaldi M et al. Percutaneous mitral repair with the MitraClip system: Safety and midterm durability in the initial EVEREST (Endovascular Valve Edge-to-Edge REpair Study) cohort. J Am Coll Cardiol, 2009; 54: 686-694.

2. Feldman T, Wasserman HS, Herrmann HC et al. Percutaneous mitral valve repair using the edge-to-edge technique: Six-month results of the EVEREST Phase I Clinical Trial. J Am Coll Cardiol, 2005; 46: 2134-2140.

3. Whitlow PL, Feldman T, Pedersen WR et al. Acute and 12-month results with catheter-based mitral valve leaflet repair: The EVEREST II (Endovascular Valve Edge-to-Edge Repair) High Risk Study. J Am Coll Cardiol, 2012; 59: 130-139.

4. Swaans MJ, Bakker AL, Alipour A et al. Survival of transcatheter mitral valve repair compared with surgical and conservative treatment in high-surgical-risk patients. J Am Coll Cardiol Cardiovasc Interv, 2014; 7: 875-881.

5. Maeda K, Tsutamoto T, Wada A, Hisanaga T, Kinoshita M. Plasma brain natriuretic peptide as a biochemical marker of high left ventricular end-diastolic pressure in patients with symptomatic left ventricular dysfunction. Am Heart J, 1998; 135: 825-832.

6. Gobinet-Georges A, Valli N, Filliatre H, Dubernet MF, Dedeystere O, Bordenave L. Stability of brain natriuretic peptide (BNP) in human whole blood and plasma. Clin Chem Lab Med, 2000; 38: 519-523.

7. Shimizu H, Aono K, Masuta K, Asada H, Misaki A, Teraoka H. Stability of brain natriuretic peptide (BNP) in human blood samples. Clin Chim Acta, 1999; 285: 169-172.

8. Giannini C, Petronio AS, De Carlo M et al. Integrated reverse left and right ventricular remodelling after MitraClip implantation in functional mitral regurgitation: An echocardiographic study. Eur Heart J Cardiovasc Imaging, 2014; 15: 95-103. 
9. Neuss M, Schau T, Schoepp M et al. Patient selection criteria and midterm clinical outcome for MitraClip therapy in patients with severe mitral regurgitation and severe congestive heart failure. Eur J Heart Fail, 2013; 15: 786-795.

10. Seifert M, Schau T, Schoepp M, Arya A, Neuss M, Butter C. MitraClip in CRT non-responders with severe mitral regurgitation. Int J Cardiol, 2014; 177: 79-85.

11. Franzen O, van der Heyden J, Baldus S et al. MitraClip(R) therapy in patients with end-stage systolic heart failure. Eur J Heart Fail, 2011; 13: 569-576.

12. Ridker PM. Clinical application of C-reactive protein for cardiovascular disease detection and prevention. Circulation, 2003; 107: 363-369.

13. Stahli BE, Grunenfelder J, Jacobs S et al. Assessment of inflammatory response to transfemoral transcatheter aortic valve implantation compared to transapical and surgical procedures: A pilot study. J Invasive Cardiol, 2012; 24: 407-411.

14. Zoghbi WA, Enriquez-Sarano M, Foster E et al. Recommendations for evaluation of the severity of native valvular regurgitation with two-dimensional and Doppler echocardiography. J Am Soc Echocardiogr, 2003; 16: 777-802.

15. Gaemperli O, Moccetti M, Surder D et al. Acute haemodynamic changes after percutaneous mitral valve repair: Relation to midterm outcomes. Heart, 2012; 98: 126-132.
16. Frangieh AH, Gruner C, Mikulicic F et al. Impact of percutaneous mitral valve repair using the MitraClip system on tricuspid regurgitation. EuroIntervention, 2016; 11: E1680- E1686.

17. Feringa HH, Poldermans D, Klein P et al. Plasma natriuretic peptide levels reflect changes in heart failure symptoms, left ventricular size and function after surgical mitral valve repair. Int J Cardiovasc Imaging, 2007; 23: 159-165.

18. Gaemperli O, Biaggi P, Gugelmann R et al. Real-time left ventricular pressure-volume loops during percutaneous mitral valve repair with the MitraClip system. Circulation, 2013; 127: 1018-1027.

19. Franzen O, Baldus S, Rudolph V et al. Acute outcomes of MitraClip therapy for mitral regurgitation in high-surgicalrisk patients: Emphasis on adverse valve morphology and severe left ventricular dysfunction. Eur Heart J, 2010; 31: 1373-1381.

20. Auricchio A, Schillinger W, Meyer S et al. Correction of mitral regurgitation in nonresponders to cardiac resynchronization therapy by MitraClip improves symptoms and promotes reverse remodeling. J Am Coll Cardiol, 2011; 58: 2183-2189.

21. Webb JG, Pasupati S, Humphries K et al. Percutaneous transarterial aortic valve replacement in selected high-risk patients with aortic stenosis. Circulation, 2007; 116: 755-763. 